\title{
Expression of Intercellular Adhesion Molecule-1 in the Livers of Rats Treated with Diethylnitrosamine
}

\author{
Shunichi Matsuoka, Hiroshi Matsumura, Yasuo Arakawa, Hitomi Nakamura, Kazushige Nirei, \\ Hiroaki Yamagami, Masahiro Ogawa, Noriko Nakajima, Shunichi Amaki, Naohide Tanaka, \\ and Mitsuhiko Moriyama*
}

Division of Gastroenterology and Hepatology, Department of Medicine, Nihon University School of Medicine, 30-1 Oyaguchi-Kamimachi, Itabashi-ku, Tokyo 173-8610, Japan

Received 10 November, 2008; Accepted 31 January, 2009

\begin{abstract}
Summary It has been reported that levels of soluble intercellular adhesion molecule-1 (ICAM-1) in the blood are elevated in hepatocellular carcinoma patients. In the present study, serial observations of the localization of ICAM-1 in the liver were made by light and electron microscopy in rats with carcinogen-induced cancer. Male Fisher rats were given diethylnitrosamine (DEN) orally in their drinking water. Rats were sacrificed at $6,8,12$, or 14 weeks after the start of DEN administration and the liver tissue was collected. ICAM-1 expression in liver was assessed using indirect immunoperoxidase staining with anti-rat ICAM-1 antibody. Although ICAM-1 expression by endothelial cells in livers of DEN-treated rats was lower than in the control group at 8 weeks, it was higher in the membrane and cytoplasm of hepatocytes. The expression of ICAM-1 in mesenchymal cells was decreased, paralleling development of cellular atypia, whereas in hepatocyte membranes and cytoplasm it was increased in these atypia. ICAM-1 was localized to the cytoplasm of cancer cells, but to the membrane of hepatocytes in the treated livers at 14 weeks. Furthermore, the levels of ICAM-1 in mesenchymal cells tended to be lower in the cancerous area than in the atypical hyperplastic nodule, and were reduced as the density of cell atypia increased, in comparison to cells in areas without cancerous nodules. We concluded that ICAM-1 may be influenced the development of cancer induced in the rat liver by a chemical carcinogen.
\end{abstract}

Key Words: intracellular adhesion molecule-1 (ICAM-1), rat, Diethylnitrosamine (DEN), cancer, immunoperoxidase staining

\section{Introduction}

Intercellular adhesion molecule-1 (ICAM-1) is member of the immunoglobulin superfamily and a ligand for leukocyte function associated antigen 1 [1-3]. It has been reported already that levels of soluble(s) ICAM-1 in the blood correlate with the intensity of intrahepatic fibrosis in human $\mathrm{C}$-viral chronic hepatitis $(\mathrm{CH})$ or liver cirrhosis (LC) and are

\footnotetext{
*To whom correspondence should be addressed.

Tel: +81-3-3972-8111 ext. 2423 Fax: $+81-3-3956-8496$

E-mail: moriyama@med.nihon-u.ac.jp
}

elevated in hepatocellular carcinoma (HCC) [4-6]. Recently, enhanced expression of ICAM-1 has been reported in human HCC and ICAM-1 is localized in the cell membrane and endoplasmic reticulum of the tumour cells $[7,8]$. In particular, it has been reported that ICAM-1 promotes the growth of residual liver cells, improves delayed hepatic regeneration in disturbed livers, and inhibits the development of hepatic fibrogenesis or cirrhosis [9-11]. We have detected already sICAM-1 in blood and intrahepatic ICAM-1 in patients with $\mathrm{C}$-viral CH, LC, and HCC [12]. In these studies, levels of sICAM-1 in blood were significantly higher in patients with $\mathrm{HCC}$ than in chronic hepatitis and LC patients. Furthermore, we reported that measurement of sICAM-1 levels in blood 

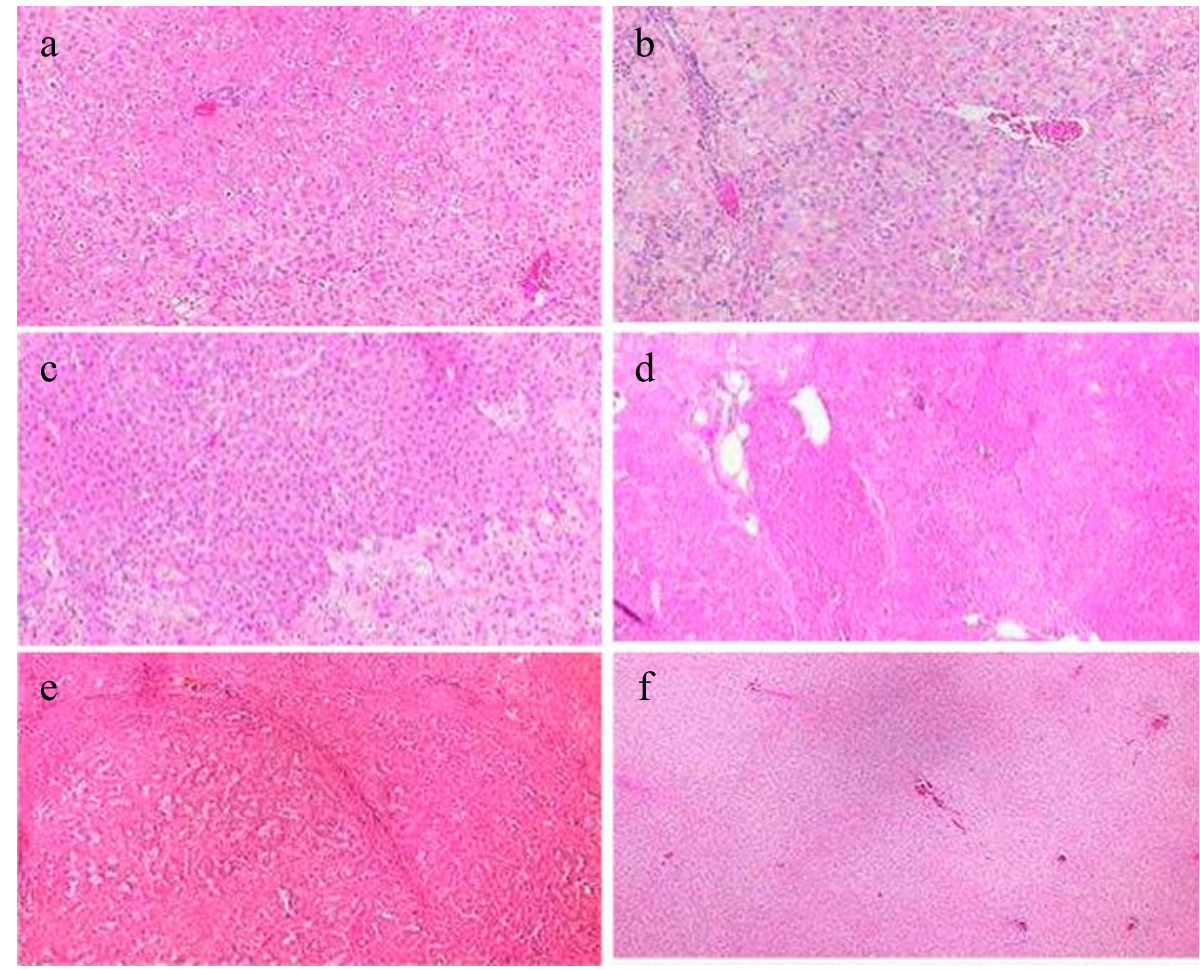

Fig. 1. Histological findings in the livers of DEN-treated and control rats. (a) Hepatocyte nuclei are irregular in size and changes in the staining of the cytoplasm are visible in some animals sacrificed at 6 weeks. (b) Distorted structure of hepatic lobules, and size variation of hepatocyte nuclei as well as a tendency to basophilic staining is increased further in some animals sacrificed at 6 weeks. (c) In some areas, groups of hepatocytes with strong atypia are present. (d) Marked nodulation observed in each animal sacrificed at 12 weeks and parts of the nodules are considered to be AH. (e) Small CA observed in all rats except for No. 13. (f) No histological changes in either control animal sacrificed at 14 weeks (Hematoxylin and eosin staining, original magnification: a, $\times 10 ; \mathrm{b}, \times 10 ; \mathrm{c}, \times 10 ; \mathrm{d}, \times 4 ; \mathrm{e}, \times 4 ; \mathrm{f}, \times 4)$.

was useful clinically for early diagnosis of HCC and decisions of efficacy of therapy in HCC patients, because ICAM-1 was expressed in the cancer cells of these patients. Therefore, in the present study, we made serial observations of the localization of ICAM-1 in liver by light and electron microscopy in rats with carcinogen-induced cancer. We sought to clarify whether ICAM-1 expression also are related to hepatocarcinogenesis in rat livers treated with a chemical carcinogen.

\section{Materials and Methods}

\section{Animals}

Male Wistar rats (200-225 g) were given Diethylnitrosamine (DEN; $100 \mathrm{mg} /$ litle Sigma Chemical Co., St. Louis, $\mathrm{MO}$ ), dissolved in their drinking water, and libitum for up to 14 weeks. Of the DEN-treated rats, 5 were sacrificed at 6 weeks (Rats No. 1 to No. 5), 4 at 8 weeks (Rats No. 6 to No. 9), 5 at 12 weeks (Rats No. 10 to No. 14), and 3 at 14 weeks (Rats No. 15 to No. 17). The control rats were given water without DEN, and one was sacrificed at each of weeks 6,8 ,
12 and 14. These experimental animals were treated in accordance with the guidelines of Nihon University (1989).

\section{Histological analysis of liver tissue}

Livers were obtained following sacrifice and divided into two parts. One part was fixed in $10 \%$ buffered formalin and embedded in paraffin and the other used for the detection of ICAM-1. Each paraffin-embedded specimen was cut into 3 to 4 micrometer sections, which were stained with hematoxylin and eosin (HE).

\section{Localization of ICAM-1 in liver}

The liver tissue was frozen rapidly with dry ice and acetone, with OCT compound for embedding, and stored at $-80^{\circ} \mathrm{C}$ until use. Four micrometer sections were fixed with acetone and subjected to two sessions of microwave treatment for $30 \mathrm{sec}$. After $20 \mathrm{~min}$ at room temperature, the ICAM-1 in liver was detected using an indirect immunoperoxidase staining method with polyclonal anti-ICAM-1 antibody (1:500 dilution) as the primary antibody and peroxidase-labeled anti-rabbit Immunoglobulin G (MBL, 

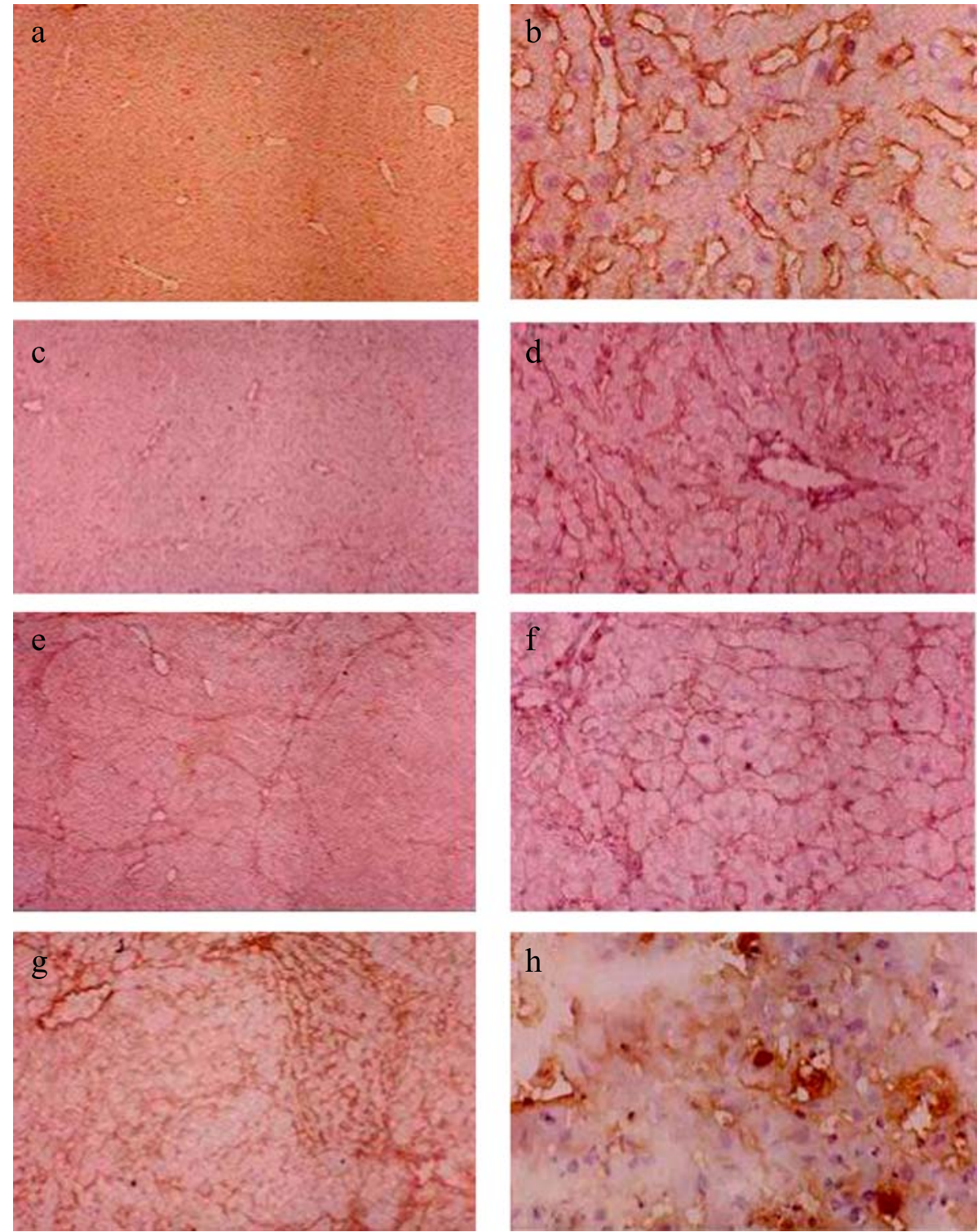

Fig. 2. Localization of ICAM-1 in the livers of rats sacrificed at each time point. Intrahepatic ICAM-1 staining is diffuse and observed predominantly in the sinusoidal endothelial cells and hepatocyte membranes of the two control animals sacrificed at each time point ( $a$ and b). The intrahepatic ICAM-1 in DEN rats sacrificed at 6 weeks is expressed at moderate levels by endothelial cells and hepatocyte membranes without cellular atypia and less strongly in the membranes of hepatocytes with cellular atypia (c and d). The expression of intrahepatic ICAM-1 in DEN rats sacrificed at 8 weeks is moderate in the endothelial cells and hepatocyte membranes without cellular atypia, and slight to moderate with cellular atypia (e and f). (g) ICAM-1 expressed at higher levels in hepatocytes with strong atypia located with a map-like distribution than in areas without cellular atypia. (f) Intrahepatic ICAM-1 in the cancerous region expressed at low levels in the cancer cell membranes of rats sacrificed at 14 weeks (Counterstained by hematoxylin, original magnification: $a, \times 4 ; \mathrm{b}, \times 20 ; \mathrm{c}, \times 4 ; \mathrm{d}, \times 10$; e, $\times 4$; $\mathrm{f}, \times 20 ; \mathrm{g}, \times 4 ; \mathrm{h}, \times 20$ ).

Tokyo, Japan, 1:300 dilution) as the secondary antibody. This investigation was carried out in animals from the DEN administration and control groups at 6, 8, 12 and 14 weeks. The degree of ICAM-1 expression in liver was semiquantitatively classified into 5 grades (none, absent; slight, presence of only several positive cells in all lobules; mild, scattered positive cells in several lobules; moderate, scattered positive cells in all lobules; marked, diffuse positive cells in all lobules).

For immunoelectron microscopy (IEM) after the 3,3'diaminobenzidine (DAB, SIGMA, St. Louis) reaction, the tissues were incubated with $2.5 \%$ glutaraldehyde (NISSIN EM Co., Ltd., Tokyo, Japan) and 2\% osmic acid (NISSIN EM Co., Ltd.) for $60 \mathrm{~min}$ at room temperature, and then 
Table 1. Intrahepatic I CAM-1 expression in Irat livers with DEN Chemostimulated Carcinogenesis.

\begin{tabular}{|c|c|c|c|c|c|c|c|c|}
\hline & \multirow{2}{*}{ Rat No. } & \multirow{2}{*}{$\begin{array}{l}\text { Mesenchymal } \\
\text { Cells }\end{array}$} & \multicolumn{2}{|c|}{ Hepatocyte } & \multicolumn{2}{|c|}{ Atypical hyperplasia } & \multicolumn{2}{|c|}{ Cancer Cell } \\
\hline & & & Membrane & Cytoplasm & Membrane & Cytoplasm & Membrane & Cytoplasm \\
\hline \multirow[t]{5}{*}{$6 w$} & 1 & modrate & none & none & n.d & n.d & n.d & n.d \\
\hline & 2 & moderate & none & none & n.d & n.d & n.d & n.d \\
\hline & 3 & moderate & slight & none & n.d & n.d & n.d & n.d \\
\hline & 4 & moderate & slight & none & n.d & n.d & n.d & n.d \\
\hline & $5(\mathrm{ct})$ & marked & none & none & n.d & n.d & n.d & n.d \\
\hline \multirow[t]{4}{*}{$8 w$} & 6 & moderate & none & none & n.d & n.d & n.d & n.d \\
\hline & 7 & mild & mild & none & n.d & n.d & n.d & n.d \\
\hline & 8 & mild & mild & none & n.d & n.d & n.d & n.d \\
\hline & $9(\mathrm{ct})$ & marked & none & none & n.d & n.d & n.d & n.d \\
\hline \multirow[t]{5}{*}{$12 \mathrm{w}$} & 10 & moderate & moderate & none & slight & mild & mild & mild \\
\hline & 11 & mild & moderate & none & mild & mild & mild & slight \\
\hline & 12 & mild & moderate & none & mild & mild & slight & mild \\
\hline & 13 & mild & mild & none & none & none & n.d & n.d \\
\hline & $14(\mathrm{ct})$ & moderate & none & none & none & none & none & none \\
\hline \multirow[t]{3}{*}{$14 w$} & 15 & mild & slight & none & slight & slight & slight & mild \\
\hline & 16 & mild & slight & none & none & slight & mild & mild \\
\hline & $17(\mathrm{ct})$ & moderate & none & none & none & none & none & none \\
\hline
\end{tabular}

ct: control, n.d: not detected

embedded in epon 812 (NISSIN EM Co., Ltd.) for $48 \mathrm{~h}$ at $60^{\circ} \mathrm{C}$. Ultra thin section were obtained using an ULTRATOME III $^{\mathrm{R}}$ (LKB, Bromma, Sweeden) and observed on a JEM-100C electron microscope (JEOL, Tokyo, Japan).

\section{Sequence analysis of ICAM-1 in the cancerous foci}

Parts of the liver, apparently including cancerous regions, were taken from two rats (Nos. 15 and 16) sacrificed at 14 weeks. The nucleotide sequences of ICAM-1 DNA in the excised tissues were determined as described below, and compared to that reported previously [13]. DNA was extracted from $100 \mathrm{mg}$ of frozen liver using DNAzol (Sanko Junyaku, Tokyo, Japan) according to the manufacturer's instructions. DNA amplification was carried out using nested PCR, generating a $1769 \mathrm{bp}$ product. The sequences of the outer primer pair were 5'-CTGCTGCCTGCACTTT GCCCT-3' [ICAM-s1; sense, nucleotide position (nt) 1-21], and 5'-GTGTCCAGGTCACGAGTTCAC-3' (ICAM-a1; antisense, nt 1831-1851), and the sequences of the inner primer pair were 5'-ACCCGTGCCAGGCCCATGCTG-3' (ICAM-s2; sense, nt 39-60), and 5'-TTAGGCCTGAGG CTACAA GTA-3' (ICAM-a2; antisense, nt 1788-1808). The first round PCR reaction contained $1 \mu \mathrm{mol}$ of each outer primer, $1 \mathrm{U}$ of Taq DNA polymerase (Boehringer Mannheim, Mannheim, Germany), $200 \mu \mathrm{l}$ M of each of the four deoxynucleotides and $1 \mathrm{x}$ Taq buffer containing $1.5 \mathrm{mM} \mathrm{MgCl}_{2}$, and was amplified for 35 cycles of $94^{\circ} \mathrm{C}$ for $40 \mathrm{~s}, 55^{\circ} \mathrm{C}$ for $40 \mathrm{~s}$ and $72^{\circ} \mathrm{C}$ for $150 \mathrm{~s}$. The second round PCR amplified
$3 \mu \mathrm{l}$ of the first PCR product using 1 pico mole of each inner primer and the same buffer and cycling conditions as in the first round reaction. The second round PCR products were sequenced as described by Shibahara et al. [14] using an ABI model 310 DNA Sequencer.

\section{Results}

\section{Histological findings}

No cancer lesions were apparent in any animal sacrificed at 6 weeks. However, hepatocyte nuclei were irregular in size and changes in the staining of the cytoplasm were observed in some animals (Fig. 1a). No atypical hyperplastic areas $(\mathrm{AH})$ and cancerous areas (CA) were found in the livers of DEN-treated rats sacrificed at 8 weeks. However, the structure of the hepatic lobules was distorted, and variations in hepatocyte nuclear dimensions and the tendency to basophilic staining were increased further (Fig. 1b). Groups of hepatocytes with strong atypia were observed in some areas (Fig. 1c). A marked nodulation was observed in each animal sacrificed at 12 weeks and parts of the nodules were considered AH (Fig. 1d). In addition, small CA were observed in all rats, except No. 13 (Fig. 1e). There were no histological changes in any animals from the control group, sacrificed at 6, 8, 12 or 14 weeks (Fig. 1f).

\section{Localization of ICAM-1 in the liver}

ICAM-1 expression in the liver was diffuse and observed 
predominantly in the sinusoidal endothelial cells and membranes of hepatocytes in the one animal of the control group sacrificed at each time point (Fig. 2a and b). ICAM-1 expression in DEN rat livers at 6 weeks was moderate in the endothelial cells and slight in the membranes of hepatocytes with atypia (Fig. 2c and d). However, the levels in both endothelial cells and hepatocyte membranes were lower than in control rats sacrificed at 6 weeks (Table 1). ICAM-1 expression in liver endothelial cells of DEN rats sacrificed at 8 weeks was slight to moderate in areas where the hepatocytes were without atypia (Fig. 2e), and only slight in areas with atypia (Fig. 2f). In particular, ICAM-1 expression was stronger in membranes of hepatocytes with strong atypia located in a map-like distribution area, than in hepatocytes without atypia (Fig. 2g). ICAM-1 expression in CA was observed in the cytoplasm and membranes of the cancer cells in rats sacrificed at 12 or 14 weeks (Fig. 2h). The expression of ICAM-1 by endothelial cells in CA was much lower than in what were considered to be normal areas of the same tissue. Moreover, ICAM-1 levels in endothelial cells tended to be lower in $\mathrm{CA}$ than in the $\mathrm{AH}$ areas and were further reduced as the density of atypical hepatocytes increased, as compared with hepatocytes in non-cancer areas. On the other hand, the expression of ICAM-1 in cell membranes and cytoplasm of cancer cells or hepatocytes with severe atypia was increased compared to hepatocytes without atypia in non-cancerous areas. In particular, the degree of expression of ICAM-1 in liver correlated with a map-like distribution or anisocytosis in irregular regeneration of hepatocytes [15-17].

\section{Ultrastructural findings}

Morphology of the tissue sections was studied by electron microscopy. ICAM-1 was detected in the membranes of mesenchymal cells and those hepatocytes with seemingly severe atypia (Fig. 3a). Furthermore, ICAM-1 was observed in the endoplasmic reticulum (ER) of cancer cells (Fig. 3b).

\section{Sequence analysis}

There were no coding changes in 3 clones isolated from rat no. 15 and another 3 from no. 16 compared to the consensus sequence [13].

\section{Discussion}

In rats with DEN-induced cancer, the pattern of expression of ICAM-1 in liver differed clearly between hepatocytes and endothelial cells in parenchyma without hepatocyte atypia and the cancerous area and hepatocytes within areas of atypia, even in the same liver. The level of endothelial cell ICAM-1 expression decreased as the degree of cellular atypia increased, whereas, in contrast, it was increased in hepatocyte membranes. In particular, the
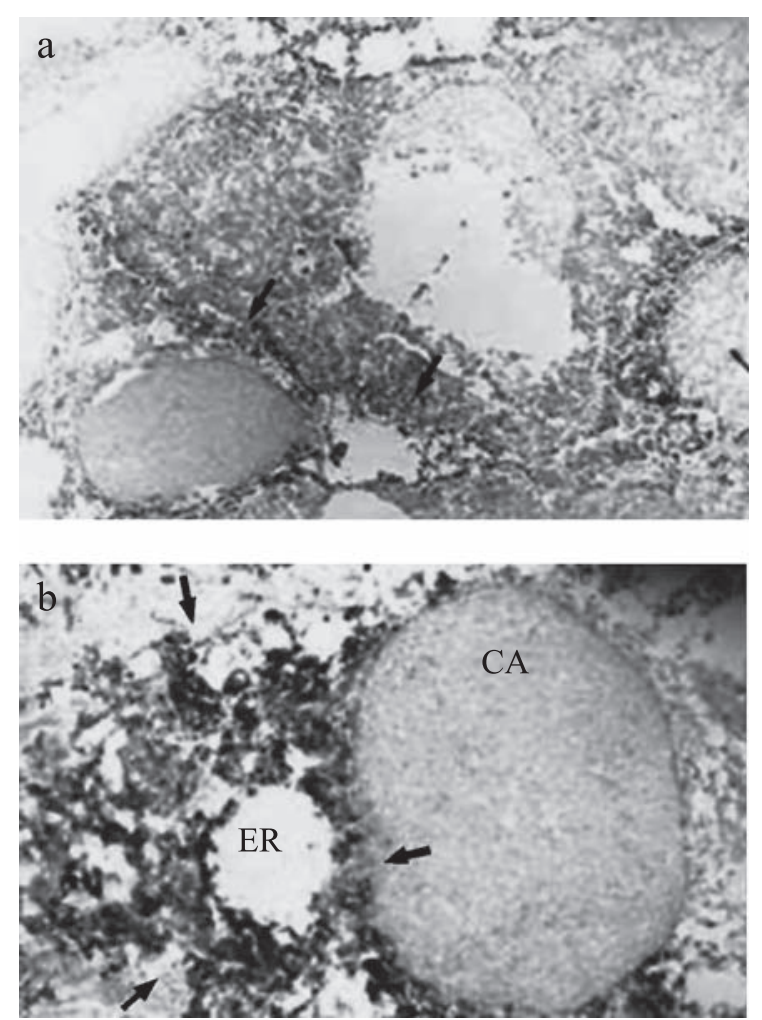

Fig. 3. (a) ICAM-1 detected by electron microscopy in the membranes of infiltrating cells, which seem to be endothelial cells, and in the membranes of hepatocytes (arrows). (b) ICAM-1 observed in the endplasmic reticulum (ER; arrows) of cancer cells (CA) (Ultra thin section EM: a, ×6,000; b, ×12,000).

expression of ICAM-1 with a map-like distribution of irregular regeneration of hepatocytes was higher than in other areas not showing this pattern. Furthermore, the expression of ICAM-1 in cell membranes and cytoplasm of cancer cells in the CA and hepatocytes in the $\mathrm{AH}$ areas was clearly greater than in areas lacking $\mathrm{CA}$ and $\mathrm{AH}$. Thus, ICAM-1 density in cancer cells and hepatocytes with severe atypia was increased more than in areas without CA or severe hepatocyte atypia. Therefore, there is a clear distinction between areas with or without hepatocyte atypia and $\mathrm{CA}$ and $\mathrm{AH}$. Furthermore, the cancer cells themselves produced the ICAM-1, because it was observed by IEM in their ER and in hepatocytes within areas of atypia. The role of ICAM-1 in hepato-carcinogenesis was not clear from the morphological observations above, but it was possible to distinguish areas with marked cellular atypia from normal areas. From these results, we consider that ICAM-1 influenced the development of hepatocyte atypia. Levels of sICAM-1 are increased parallel to development of F stages and $\mathrm{HCC}$ in type $\mathrm{C}$ liver diseases. In the present study, the expression of ICAM-1 in mesenchymal cells decreased as 
cellular atypia increased. Reciprocally, it increased in the membrane and cytoplasm of hepatocytes, paralleling the development of cellular atypia. Furthermore, the ICAM-1 was present in both the cell membrane and cytoplasm of cancer cells. The degree of expression of ICAM-1 in mesenchymal cells in CA was clearly decreased compared to the non cancerous areas of the same rat liver. Therefore, the reason that SICAM-1 concentration in humans is reported to increase as F stages or HCC develop is suggested to be that ICAM-1 secretion by hepatocytes with atypia or by cancer cells is increased.

In addition, we analyzed the gene sequence of ICAM-1 from parts of livers including CA two different rats (Nos. 15 and 16) sacrificed at 14 weeks. The nucleotide sequences of ICAM-1 DNA from the excised tissues were determined and compared with that reported previously [13]. This analysis showed no coding changes in CA from rat liver relative to the consensus sequence. This indicated that ICAM-1 secreted by the cancer cells was homogeneous and the cells did not produce a variant molecule. Therefore, we consider that variant ICAM-1 does not participate in the progression of the liver fibrosis or hepatocarcinogenesis.

What are the factors which promote the expression of atypia of hepatocytes in liver? Some factors have been identified whose serum levels increase in parallel to the development of $\mathrm{F}$ stage in chronic hepatitis and liver cirrhosis and some factors are present at high levels in patients with HCC. Such factors include, ICAM-1 [4-6], human hepatocyte growth factor (HGF) [18, 19], vascular endothelial growth factor [20] and others. It is thought that these factors are produced and secreted by hepatoma cells. It has been confirmed that HGF is expressed by hepatocytes with strong atypia in our previous studies [21, 22]. In this study, we also confirm that strong expression of ICAM-1 is found in hepatocytes with strong atypia, decreased expression of ICAM-1 is shown in endothelial cells is shown in $\mathrm{AH}$ and small CA. Therefore, it is suggested that ICAM- 1 is produced and secreted by these cells. Although it was not clear from our present study, we suggest that ICAM-1 influences the development of atypia of hepatocytes by one of the mechanisms mentioned above.

\section{References}

[1] Marlin, S.D. and Springer, T.A.: Purified intercellular adhesion molecule-1 (ICAM-1) is a ligand for lymphocyte function-associated antigen 1 (LFA-1). Cell, 51, 813-819, 1987.

[2] Becker, J.C., Dummer, R., Hartmann, A.A., Burg, G., and Schmidt, R.: Shedding of ICAM-1 from human melanoma cell lines induced by IFN- $\gamma$ and tumor necrosis factor- $\alpha$. $J$. Immunol., 147, 4398-4401, 1991.

[3] Rothlein, R., Mainolfi, E.A., Czajkowski, M., and Marlin,
S.D.: A form of circulating ICAM-1 in human serum. $J$. Immunol., 147, 3788-3793, 1991.

[4] Zohrens, G., Armbrust, T., Pirzer, U., Meyer zum Buschenfelde, K.H., and Ramadori, G.: Intercellular adhesion molecule-1 concentration in sera of patients with acute and chronic liver disease: relationship to disease activity and cirrhosis. Hepatology, 18, 798-802, 1993.

[5] Shimizu, Y., Minemura, M., Tsukishiro, T., Kashii, Y., Miyamoto, M., Nishimori, H., Higuchi, K., and Watanabe, A.: Serum concentration of intercellular adhesion molecule-1 in patients with hepatocellular carcinoma is a marker of the disease progression and prognosis. Hepatology, 22, 525-531, 1995.

[6] Hyodo, I., Jinno, K., Tanimizu, M., Hosokawa, Y., Nishikawa, Y., Akiyama, M., Mandai, K., and Moriwaki, S.: Detection of circulating intercellular adhesion molecule-1 in hepatocellular carcinoma. Int. J. Cancer, 55, 775-779, 1993.

[7] Momosaki, S., Yano, H., Ogasawara, S., Higaki, K., Hisaka, T., and Kojiro, M.: Expression of intercellular adhesion molecule 1 in human hepatocellular carcinoma. Hepatolog., 22, 1708-1713, 1995.

[8] Torii, A., Harada, A., Nakao, A., Nonami, T., Ito, M., and Takagi, H.: Expression of intercellular adhesion molecule-1 in hepatocellular carcinoma. J. Surg. Oncol., 53, 239-242, 1993.

[9] Hellerbrand, C., Wang, S.C., Tsukamoto, H., Brenner, D.A., and Rippe, R.A.: Expression of intracellular adhesion molecule 1 by activated hepatic stellate cells. Hepatology, 24, 670-676, 1996.

[10] Hellerbrand, C., Jobin, C., Iimuro, Y., Licato, L., Sartor, R.B., and Brenner, D.A.: Inhibition of NF kappa B in activated rat hepatic satellite cells by proteasome inhibitors and an I kappa B super-repressor. Hepatology, 27, 12851295, 1998.

[11] Kobayashi, H., Horikoshi, K., Long, L., Yamataka, A., Lane, G.J., and Miyano, T.: Serum concentration of adhesion molecules in postoperative biliary atresia patients: Relationship to disease activity and cirrhosis. J. Pediatr. Surg., 36, 1297-1301, 2001.

[12] Moriyama, M., Matsumua, H., Shioda, J., Aoki, H., Nakamura, H., Arakawa, Y., Neirai, K., Yamagami, H., Kaneko, M., Tanaka, N., and Arakawa, Y.: Measurement of human intercellular adhesion molecule 1 in the blood is useful for predicting the occurrence of hepatocellular carcinomas from chronic hepatitis $\mathrm{C}$ and liver cirrhosis. Intervirology, 49, 327-338, 2006.

[13] Kita, Y., Takashi, T., Iigo, Y., Tamatani, T., Miyasaka, M., and Horiuchi, T.: Sequence and expression of rat ICAM-1. Biochim. Biophys. Acta, 1131, 108-110, 1992.

[14] Shibahara, N., Moriyama, M., Abe, K., Tanaka, N., and Arakawa, Y:: Biochemicaland virological response to interferon therapy in patioents with chronic hepatitis C, coinfected with hepatitis G virus. J. Viral. Hepat., 7, 43-50, 2000 .

[15] Uchida, T.: Small hepatocellular carcinoma: Its relationship to multistep hepatocarcinogenesis. Pathol. Int., 45, 175-184, 1995. 
[16] Uchida, T.: Pathology of Hepatitis C. Intervirology, 37, 126132, 1994.

[17] Ueno, Y., Moriyama, M., Uchida, T., and Arakawa, Y.: Irregular regeneration of hepatocytes is an important factor in the hepatocarcinogenesis of liver disease. Hepatology, 33, 357-362, 2001.

[18] Tsubouchi, H., Niitani, Y., Hirono, S., Nakayama, H., Gohda, E., Arakaki, N., Sakiyama, O., Takahashi, K., Kimoto, M., Kawakami, S., Setoguchi, M., Tachikawa, T., Shin, S., Arima, T., and Daikuhara, Y.: Levels of the human hepatocyte growth factor in serum of patients with various liver diseases determined by an enzyme-linked immunosorbent assay. Hepatology, 13, 1-5, 1991.

[19] Yamagami, H., Moriyama, M., Tanaka, N., and Arakawa, Y.: Detection of serum and intrahepatic human hepatocyte growth factor in patients with C-viral liver diseases. Intervirology, 44,
36-42, 2001.

[20] Poon, R.T., Ng, I.O., Lau, C., Zhu, L.X., Yu, W.C., Lo, C.M., Fan, S.T., and Wong, J.: Serum vascular endothelial growth factor predicts venous invasion in hepatocellular carcinoma: a prospective study. Ann. Surg., 233, 227-235, 2001.

[21] Kanda, K., Moriyama, M., Shibahara, N., Shimizu, Y., Segawa, Y., Matsumura, H., Kaneko, M., Shioda, A., Komine, F., Suzuki, K., Tanaka, N., Okubo, H., and Arakawa, Y.: Expression of HGF mRNA during rat chemically induced hepatocarcinogenesis. Gastroenterology (suppl), 112, A1297, 1997.

[22] Okubo, H., Moriyama, M., Tanaka, N., and Arakawa, Y.: Detection of serum and intrahepatic hepatocyte growth factor during DEN-induced carcinogenesis in the rat. Hepatol. Res., 24, 385-394, 2002. 